\title{
WCC 2017-A-112: CT ANGIOGRAPHIC ASSESSMENT OF CORONARY ARTERY BYPASS GRAFT
}

\author{
Abhishek Mashirkar, Bhwana D. Sonawane
}

\begin{abstract}
:
Introduction: CT Angiography (CTA) is a noninvasive imaging modality to evaluate the vascular system. In coronary artery bypass graft patients, this study is helpful to evaluate the status of graft.
\end{abstract}

\section{AIMS \& OBJECTIVE:}

To evaluate the coronary artery bypass graft on CT angiography.

\section{MATERIAL \& METHOD:}

This was a prospective study conducted during Feb-15 to April-16 at our institute. Total 91 post-CABG patients referred from CVTS department were undergone CT angiography study using standard CTA-CABG protocol on 128 slice CT scanner. Post processing was done and images were analyze for presence and patency of graft, distal native artery status.

\section{RESULT:}

Total 137 grafts were evaluated in 91 patients of which $73 \%$ were patent and $27 \%$ were stenosed/occluded. 90 were arterial grafts of which $27.77 \%$ were stenosed/occluded. Out of total 47 venous grafts, $25.53 \%$ showed stenosis/occlusion. Distal native artery was normal in $68.61 \%$ cases and in rest cases it showed stenosis/occlusion.

\section{CONCLUSION:}

CTA noninvasively assess the presence and patency of the graft and distal native artery status in post-CABG patients, which guides the CVTS surgeon in the management of these patients. Our study showed no significant difference in the type of graft (arterial/venous) and their stenosis/occlusion.

Article received on 01 JAN 2017, published on 31JAN 2017.

Abhishek Mashirkar ${ }^{1}$, Bhwana D. Sonawane ${ }^{2}$

1Senior Resident, Radiology, Dept GMCH \& SSH Nagpur, Maharashtra.

2Professor \& HOD, Radiology, GMC \& SSH Nagpur, Maharashtra.

Corresponding author: Abhishek Mashirkar,

Email: dr_abhi007@yahoo.com 